\title{
Asociación de lesiones bucales con el estado serológico para el VIH
}

Velia Ramírez-Amador, CD, MSC, PhD, (1) Lilly Esquivel-Pedraza, CD, (2) Esther Irigoyen-Camacho, CD, MPH, D ra en C ${ }^{(1)} \mathrm{G}$ abriela A naya-Saavedra, CD, (1) Imelda G onzález-Ramírez, CD. ${ }^{(1)}$

Ramírez-Amador V, Esquivel-Pedraza L, Irigoyen-Camacho E,Anaya-Saavedra G, González-Ramírez I. Asociación de lesiones bucales con el estado serológico para el VIH. Salud Publica Mex 2002;44:87-91. El texto completo en inglés de este artículo está disponible en: http://www.insp.mx/salud/index.html

\section{Resumen}

Objetivo. Estimar la prevalencia de lesiones bucales y su asociación con el estado serológico del VIH. Material y métodos. Estudio transversal, descriptivo y doble ciego, hecho, entre 1998 y 1999 en México, D.F., con 512 personas que acudieron a dos centros de información del Centro N acional para la Prevención delVIH/SIDA e ITS (CO N ASIDA) y a quienes se les practicó examen bucal sin conocer aún su estado serológico con respecto alVIH. Se utilizaron las pruebas de t-Student, exacta de Fisher y $\chi^{2}$. Se calculó la razón de productos cruzados. Resultados. Se examinaron 512 individuos, 68 resultaron positivos al VIH. En 65\% (44/68), se observaron lesiones bucales asociadas con el VIH (LB), 95\% correspondieron a candidosis bucal (CB) y leucoplasia vellosa (LV). La asociación de CB y LV con el estado serológico positivo al VIH fue estrecha. Conclusiones La CB y la LV fueron las lesiones más fuertemente asociadas con el VIH. El texto completo en inglés de este artículo está disponible en: http://www.insp.mx/salud/ index.html

Palabras clave:VIH/SIDA; lesiones bucales; candidosis bucal; leucoplasia vellosa; México

\author{
Ramírez-Amador V, Esquivel-Pedraza L, \\ Irigoyen-Camacho E,Anaya-Saavedra G, \\ González-Ramírez I. \\ Association of oral lesions \\ with HIV serological status. \\ Salud Publica Mex 2002;44:87-91. \\ The English version of this paper \\ is available at: http://www.insp.mx/salud/index.html
}

\section{Summary}

Objective To estimate the prevalence of oral lesions and its association with HIV serological status. Material and MethodsA cross-sectional descriptive study was conducted between 1998 and 1999 in Mexico C ity, among 512 subjects attending two information centers of $\mathrm{C}$ entro $\mathrm{N}$ acional para la Prevención del VIH/SIDA e ITS (CONASIDA, National Center for Prevention of HIV/AID S and SexuallyTransmitted Infections) for HIV serologic testing. The oral examination was performed without knowledge of the HIV status. Statistical analysis was conducted using Student's t test, Fisher's exact test and the $\chi^{2}$ test; 0 dds ratios and $95 \% \mathrm{Cl}$ were also calculated. Results. $A$ to tal of 512 individuals were examined, 68 of whom were HIV-positive. HIV-related oral lesions (O L), were evident in 65\% (44/68) of the HIV-positive individuals; $95 \%$ of them consisted in oral candidosis (OC) and hairy leukoplakia (HL).OC and $\mathrm{HL}$ were strongly asso ciated with seropositivity to HIV.Conclusions $\mathrm{OC}$ and HL were the oral lesions most strongly associated to HIV seropositivity. The English version of this paper is available at: http://www.insp.mx/salud/index.html

Key words:HIV/AIDS; oral lesions; oral candidosis; hairy leukoplakia; Mexico
E $\mathrm{n}$ los pacientes infectados por el virus de inmuno E deficiencia humana (VIH), las manifestaciones bucales asociadas con esta infección juegan un papel importante debido a la elevada frecuencia con la que se presentan, que varía entre $37 \%$ y $60 \%$, en diferentes series, ${ }^{1-2}$ así como por el deterioro en la calidad de vida que algunas lesiones bucales, tales como las úlceras recurrentes, la periodontitis ulceronecrosante y las lesiones avanzadas de sarcoma de Kaposi pueden causar al afectar funciones como la deglución y la mastica-

(1) Universidad Autónoma Metropolitana-X ochimilco, México, D.F., México.

(2) Instituto $\mathrm{N}$ acional de Ciencias Médicas y de la N utrición "Salvador Zubirán", México, D.F., México.

Fecha de recibido: 15 de febrero de 2001 • Fecha de aprobado: 19 de octubre de 2001 Solicitud de sobretiros:Velia Ramírez-Amador. Camino Sta.Teresa 277-9, 14010 México, D.F., México. Correo electrónico: rava1863@ cueyatl.uam.mx 
ción. $\cdot^{3-6}$ La candidosis bucal (CB) y la leucoplasia vellosa (LV) son consideradas indicadoras de la infección por el VIH, signos de inmunosupresión profunda, de carga viral elevada y de progresión de la enfermedad en los individuos seropositivos a este virus..$^{7-11}$

En México, algunos estudios han descrito la prevalencia y las características clínicas de las alteraciones bucales asociadas con el VIH. ${ }^{12-14}$ No obstante, la prevalencia de lesiones bucales y sus características clínicas, al momento de la detección de un paciente con el VIH, aún no ha sido descrita en un estudio con método de doble ciego.

El objetivo del presente estudio fue el de estimar la prevalencia de las alteraciones bucales y su posible asociación con el estado serológico para el VIH, al momento del diagnóstico de la infección, en individuos que acuden a los centros de información del CONASIDA en la ciudad de México.

\section{Material y métodos}

Se realizó un estudio transversal, descriptivo y doble ciego, en dos centros de información del CONASIDA (Flora y Tlalpan), localizados en la Ciudad de México en donde se efectuaron exámenes de la cavidad bucal en individuos mayores de 15 años, que acudieron de manera consecutiva a solicitar el examen serológico para la detección de anticuerpos contra el VIH y que aceptaron participar en el estudio bajo consentimiento informado por escrito. El estudio se efectuó en dos periodos: dos semanas en junio de 1998 y dos semanas en junio de 1999.

Se utilizó un cuestionario que comprendió datos demográficos y hábitos de consumo de tabaco y alcohol. Estos últimos se incluyeron, por ser considerados como posibles variables confusoras, debido a la asociación que se ha observado entre estos factores y la presencia de lesiones bucales independientemente del estado serológico. ${ }^{4,15-17} \mathrm{Al}$ momento de realizar el cuestionario y la inspección bucal, el estado serológico de los participantes era desconocido tanto para el examinador como para el examinado.

Para el diagnóstico de cada una de las lesiones bucales se siguieron los criterios clínicos descritos en la Clasificación Internacional de las lesiones bucales asociadas con la infección por el VIH. ${ }^{18}$ De acuerdo con estos criterios clínicos, los ocho examinadores participantes llevaron a cabo ejercicios de inter e intra-calibración, hasta obtener niveles aceptables $(k=0.63)$. Las cuatro especialistas que participaron en la supervisión de los examinadores obtuvieron un porcentaje de acuerdo intra-examinador superior a $96 \%(k=0.97)$, e inter-examinador de entre 80 y $95 \%(k=0.73)$.
Los resultados del estado serológico a la infección por el VIH y la información sobre las prácticas de riesgo se obtuvieron de los expedientes clínicos. En aquellos sujetos que resultaron con el VIH, se realizó una segunda revisión de expedientes con la finalidad de obtener el recuento de linfocitos $\mathrm{CD} 4^{+}$.

Se obtuvo la prevalencia de cada una de las lesiones bucales asociadas con el VIH de acuerdo con el estado serológico de los sujetos. Se estimaron las medias y desviaciones estándar $(D E)$ de las variables continuas y las proporciones para las variables discretas. Para la comparación de las características demográficas y clínicas, así como de las lesiones bucales de los individuos positivos al VIH con los negativos, se utilizó la prueba de $t$-Student (no pareada), para las variables continuas. Se empleó la prueba de $\chi^{2}$ de Pearson para estudiar la relación entre el estado serológico de los participantes y la presencia de lesiones de la mucosa bucal. Cuando los valores esperados por celda eran menores a cinco se utilizó la prueba exacta de Fisher. Asimismo, se calculó la razón de productos cruzados (RPC) y sus intervalos de confianza (IC 95\%) para analizar esta asociación.

\section{Resultados}

Un total de 512 personas participaron en el estudio, de las cuales 228 fueron examinadas en el primer corte transversal y 284 en el segundo (cuadro I). No se encontraron diferencias estadísticamente significativas entre los dos cortes transversales en relación con las variables consideradas en el estudio (edad, género, uso de tabaco o alcohol, categoría de transmisión, linfocitos $\mathrm{CD}^{+}$y presencia de lesiones asociadas con el VIH), por lo que los resultados se presentan como una sola población.

La prevalencia de las lesiones bucales asociadas con el VIH (LB) fue de (44/68) $64.7 \%$ en las personas VIH positivas, mientras que en el grupo de personas VIH negativas fue del $(14 / 444) 3.1 \%(p<0.01)$. En $42(95.4 \%)$ de las 44 personas con el VIH y lesiones bucales, se presentaron lesiones altamente asociadas con el VIH; en 34.1\% (15/44) de los sujetos, las lesiones se observaron de manera concurrente. La asociación entre las LB altamente asociadas con el VIH y el estado serológico fue estrecha, particularmente para la $\mathrm{CB}$, en sus dos variedades, y para la LV (cuadro II).

Cinco de los individuos seropositivos al VIH, y ninguno de los seronegativos, presentaron enfermedades periodontales asociadas con el $\mathrm{VIH}$, cuatro con eritema lineal gingival y uno con gingivitis ulceronecrosante $(p<0.01)$. Otras LB, como herpes labial, úlceras recurrentes y xerostomía, no mostraron dife- 


\section{Cuadro I}

\section{Características demográficas y clínicas de la muestra. México, D.F., 1998-1999}

\begin{tabular}{|c|c|c|c|c|c|c|c|c|c|c|c|c|}
\hline & \multicolumn{4}{|c|}{$\begin{array}{c}\text { Estudio } 1998 \\
\quad(n=228)\end{array}$} & \multicolumn{4}{|c|}{$\begin{array}{l}\text { Estudio } 1999 \\
\quad(n=284)\end{array}$} & \multicolumn{4}{|c|}{$\begin{array}{c}\text { Total } \\
(n=512)\end{array}$} \\
\hline & \multicolumn{2}{|c|}{$\mathrm{VIH} \cdot(n=194)$} & \multicolumn{2}{|c|}{$\mathrm{VIH}^{+}(\mathrm{n}=34)$} & \multicolumn{2}{|c|}{$\mathrm{VIH} \cdot(n=250)$} & \multicolumn{2}{|c|}{$\mathrm{VIH}^{+}(\mathrm{n}=34)$} & \multicolumn{2}{|c|}{$\mathrm{VIH}^{-}(n=444)$} & \multicolumn{2}{|c|}{$\mathrm{VIH}^{+}(\mathrm{n}=68)$} \\
\hline & $n$ & $\%$ & $n$ & $\%$ & $n$ & $\%$ & $n$ & $\%$ & $\mathrm{n}$ & $\%$ & $n$ & $\%$ \\
\hline \multicolumn{13}{|l|}{ Género } \\
\hline Masculino & 110 & $(56.7)$ & 29 & $(85.3)$ & 145 & $(58.0)$ & 32 & $(94.1)$ & 255 & $(57.4)$ & 61 & (89.7) \\
\hline Femenino & 84 & $(43.3)$ & 5 & $(14.7)$ & 105 & $(42.0)$ & 2 & $(5.9)$ & 189 & $(42.6)$ & 7 & $(10.3)$ \\
\hline \multicolumn{13}{|l|}{ Edad (media) } \\
\hline Años & \multicolumn{2}{|c|}{$26.8 \pm 9.1$} & \multicolumn{2}{|c|}{$31.8 \pm 9.6$} & \multicolumn{2}{|c|}{$27.4 \pm 8.8$} & \multicolumn{2}{|c|}{$31.5 \pm 8.1$} & \multicolumn{2}{|c|}{$27.2 \pm 8.9$} & \multicolumn{2}{|c|}{$31.2 \pm 8.9$} \\
\hline Lesiones bucales asociadas con el VIH ${ }^{14}$ & $14 \quad 5$ & 2.6 & 23 & 67.6 & 9 & 3.6 & 21 & 61.8 & 14 & 3.1 & 44 & 64.7 \\
\hline \multicolumn{13}{|l|}{ Categorías de transmisión } \\
\hline Homo y bisexualidad & & & 22 & $(64.7)$ & & & 24 & $(70.6)$ & & & 46 & $(67.6)$ \\
\hline Heterosexualidad & & & 9 & $(26.5)$ & & & 9 & $(26.5)$ & & & 18 & $(26.5)$ \\
\hline Vía sanguínea & & & 3 & $(8.8)$ & & & 1 & $(2.9)$ & & & 4 & $(5.9)$ \\
\hline Linfocitos CD $4^{+}$ & \multicolumn{4}{|c|}{$(n=13)$} & \multicolumn{4}{|c|}{$(n=11)$} & \multicolumn{4}{|c|}{$(n=24)$} \\
\hline (mediana) & \multicolumn{4}{|c|}{154 (intervalo 10-359) } & \multicolumn{4}{|c|}{198 (intervalo 9-560) } & \multicolumn{4}{|c|}{160 (intervalo 9-560) } \\
\hline
\end{tabular}

\section{Cuadro II}

Asociación entre El ESTAdo SEROLógico

PARA EL VIH Y EL TIPO DE LESIÓN BUCAL. Méxıco, D.F., 1998-1999

\begin{tabular}{|c|c|c|c|c|c|c|c|}
\hline & & $\begin{array}{l}\text { (IH- } \\
\text { :444) }\end{array}$ & & $\begin{array}{l}\begin{array}{l}\mathrm{H}^{+} \\
=68)\end{array}\end{array}$ & & & \\
\hline Lesiones asociadas con elVIH & $n$ & $\%$ & $\mathrm{n}$ & $\%$ & RPC & IC 95\% & $p$ \\
\hline Candidosis & 5* & (1.1) & $26^{\ddagger}$ & $(38.2)$ & 54.4 & $(20-149)$ & $<0.01$ \\
\hline Candidosis eritematosa & 4 & $(0.9)$ & 21 & $(30.9)$ & 49.1 & $(24-100)$ & $<0.01$ \\
\hline $\begin{array}{l}\text { Candidosis pseudd } \\
\text { membranosa }\end{array}$ & 2 & $(0.4)$ & 14 & $(20.6)$ & 57.3 & $(23-140)$ & $<0.01$ \\
\hline
\end{tabular}

\begin{tabular}{llcccccc} 
Q ueilitis comisural & 6 & $(1.4)$ & 7 & $(10.3)$ & 8.4 & $(2-22)$ & $<0.01$ \\
\hline Leucoplasia vellosa & 0 & $(0)$ & 21 & $(30.9)$ & 62.0 & $(16-264)$ & $<0.01$
\end{tabular}

* Un paciente con ambos tipos de candidosis

₹ Nueve pacientes con ambos tipos de candidosis

rencias significativas entre los dos grupos $(p>0.05)$. Asimismo, no se observaron diferencias con la edad entre la presencia de LB, el género, la categoría de transmisión, el consumo de alcohol, o de tabaco $(p>0.05)$.

En el presente trabajo únicamente fue posible recuperar información sobre el conteo de $\mathrm{CD}^{+}$en $35 \%$ de las personas con el VIH, debido a que la mayoría de los sujetos no regresan con estos resultados de laboratorio a CONASIDA. Considerando que la validez interna de los resultados del conteo de $\mathrm{CD}^{+}{ }^{+}$en el grupo de estudio es relativa, éstos no fueron analizados en relación con la presencia de las lesiones bucales observadas.

\section{Discusión}

Dentro de las particularidades metodológicas del presente estudio está la posible reducción de sesgos en la detección de las LB, debido a que los individuos incluidos fueron sujetos que desconocían su estado serológico para el VIH, y no eran pacientes con el VIH remitidos a centros de referencia para el diagnóstico de lesiones bucales, ni eran población hospitalaria. Por otra parte, la detección de lesiones bucales se llevó a cabo por personal especializado en patología bucal, con experiencia previa en la detección de lesiones asociadas con el VIH. Por lo tanto, la frecuencia de LB encontrada en esta serie pudiera tener mayor confiabilidad que aquellos estudios en donde se conoce el estado serológico para el VIH del sujeto, o en donde los exámenes bucales no fueron practicados por especialistas.

En este estudio, dos terceras partes (65\%) de los individuos con resultado positivo para el VIH mos- 
traron una o más de una LB habiendo en $95 \%$ de los casos lesiones altamente asociadas con el VIH. Estos hallazgos son consistentes con investigaciones previas $^{8,19-21}$ que han sugerido que la candidosis eritematosa, la candidosis pseudomembranosa y la LV, sean consideradas como marcadoras de la infección por el VIH en la población adulta.

En diversos trabajos se ha demostrado que la presencia de CB o LV, independiente o asociada, se encuentra significativamente relacionada con inmunosupresión profunda para la infección por el $\mathrm{VIH} .{ }^{11,22,23}$ Asimismo, se ha observado que la coexistencia de estas lesiones se asocia de manera más estrecha con inmunosupresión que la presencia independiente de una de ellas. ${ }^{23,}{ }^{24} \mathrm{La}$ elevada frecuencia de una o más lesiones bucales encontradas podría sugerir que una proporción importante de los individuos con el VIH examinados se encontraba en etapas avanzadas de la enfermedad.

Los sujetos con el VIH mostraron una probabilidad 50 veces mayor de presentar LV o CB que los sujetos seronegativos. Este dato resulta particularmente significativo, considerando que existe un número importante de personas en la Ciudad de México con el VIH que desconocen su estado serológico, en las cuales la detección de estas lesiones podría sugerir la práctica de pruebas para la determinación del estado serológico para el VIH, y en quienes posiblemente este tipo de lesiones pasan inadvertidas.

En conclusión, los hallazgos del presente estudio confirman la utilidad del diagnóstico de CB y LV como lesiones indicadoras de la infección por el VIH. La identificación de estas lesiones puede ser el primer paso que promueva la realización de pruebas diagnósticas y la detección temprana de pacientes con el VIH. Es importante subrayar el valor de un cuidadoso examen bucal en el diagnóstico de la infección por el VIH, ya que la exploración de los tejidos bucales es un procedimiento sencillo, de bajo costo y no invasivo.

\section{A gradecimientos}

Agradecemos sinceramente a las Directivas de CONASIDA: Dra. Patricia Uribe Zúñiga (Directora General), a la Dra. Griselda Hernández Tepichin (Directora Técnica), y al Dr. Carlos Magis Rodríguez (Director de Investigación), las facilidades brindadas para realizar el presente estudio. Asimismo, agradecemos a las doctoras Estela de la Rosa-García y Martha GonzálezGuevara, profesoras del Curso de Especialización en Diagnóstico Integral y Patología Bucal (DIPB), de la Universidad Autónoma Metropolitana-Xochimilco, y a los alumnos del mismo curso: Jesús Maldonado Mayo, Patricia Rubio Romero, Ivonne Moreno Germán,
Ariel Páez Huerta, Diana Huerta Platas, Xóchitl Gallegos Medellín, Josefina Alvarez Z, Diana Díaz R, Elizabeth Garduño G, Rebeca Guzmán M, Gloria Niño H, Saúl Sánchez Z, Marco A. Torres T, y Edgar Zaldívar S, su colaboración en la realización de este estudio.

\section{Referencias}

1. Patton LL, McKaig R, Strauss R, Rogers D, Eron J]. Changing prevalence of oral manifestations of human immunodeficiency virus in the era of protease inhibitor therapy. 0 ral Surg 0 ral Med 0 ral Pathol 0 ral Radiol Endod 2000;89:299-304.

2.ArendorfTM, Bredekamp B, Cloete CAC, Sauer G. 0 ral manifestations of HIV infection in 600 South African patients. J O ral Pathol Med 1998; 27:176-179.

3. Ficarra G 0 ral ulcers in HIV-infected patients:An update on epidemiology and diagnosis. 0 ral D is 1997;3 Suppl 1:183-189.

4. Muzyka BC, Glick M. Major aphthous ulcers in patients with HIV disease. 0 ral Surg 0 ral Med O ral Pathol 1994;77:116-120.

5. G lick M, Muzyka BC, Salkin LM, Lurie D. N ecrotizing ulcerative periodontitis: A marker for immune deterioration and a predictor for the diagnosis of AIDS.J Periodontol 1994;65:393-397.

6. Epstein JB. Management of oral Kaposi's sarcoma and a proposal for clinical staging. 0 ral D is 1997;3:S124-S128.

7. Ramírez-Amador V, Esquivel-Pedraza L, Sierra-Madero J, Soto-Ramírez L, González-Ramírez I, A naya-Saavedra $\mathrm{G}$ et al. 0 ral clinical markers and viral load in a prospective cohort of Mexican HIV-infected patients. AIDS 2001;15: 1910-1911.

8. Greenspan D, Komaroff E, Redford M, Phelan JA,N avazesh M,Alves MEAF et al. 0 ral mucosal lesions and HIV viral load in the women's interagency HIV study (W IHS). J A cquir Immune D efic Syndr 2000;25:44-50.

9. MargiottaV, C ampisi G, Mancusso S, Accurso V, A bbadessa V. HIV infection: O ral lesions, CD 4 cell count and viral load in an Italian study population. J O ral Pathol Med 1999;28:173-177.

10. Patton LL, MCKaig RG, Eron Jr JJ, Lawrence HP, Strauss RP. 0 ral hairy leukoplakia and oral candidiasis as predictors of HIV viral load. AIDS 1999;13:2174-2176.

11. Greenspan JS. Sentinels and signposts: The epidemiology and significance of the oral manifestations of HIV disease. 0 ral D is 1997;3 Suppl 1:13-17.

12. Ramírez-Amador V, Esquivel-Pedraza L, Ponce de León S, Ponce de León S. Prognostic value of oral candidosis and hairy leukoplakia in 111 Mexican HIV-infected patients. J 0 ral Pathol Med 1996;25:206-211.

13. Ramírez-A mador V, Esquivel-Pedraza L, Sierra-Madero J, Ponce de León Sa, Ponce de León Se. 0 ral manifestations of HIV infection by gender and transmission category in Mexico City. J 0 ral Pathol Med 1998;27:135-140. 14. Ramírez-A mador V, González A, De la Rosa E, G onzález M, Rivera I, Hernández $\mathrm{C}$ et al. 0 ral lesions in Mexican HIV-infected patients. J 0 ral Pathol Med 1990;19:482-485.

15. Palacio H, Hilton JF, Canchola AJ, Greenspan D. Effect of cigarrette smoking on HIV-related oral lesions. J Acquir Immune Defic Syndr 1997;14:338-342.

16. Research, Science, and Therapy C ommittee of the A merican A cademy of Peridontology. Epidemiology of periodontal diseases. J Periodontol 1996;67:935-945.

17. McC arthy G M, Mackie ID, Koval J, Sandhu HS, D aley TD. Factors associated with increased frequency of HIV-related oral candidiasis. J O ral Pathol Med 1991;20:332-336. 
18. European Community. Clearinghouse on oral problems related to HIV infection and W HO collaborating centre on oral manifestations of the immunodeficiency virus. Classification and diagnostic criteria for oral lesions in HIV infection. J O ral Pathol Med 1993;22:289-291.

19. Robinson PG, Challacombe S), Sheiham A . O ral examination:A screening tool for HIV infection? Sex Transm Infect 1998;74:345-348.

20. Shiboski CH, Hilton JF, G reenspan D, W estenhouse JL, D erish P,Vranizan $\mathrm{K}$ et al. HIV-Related oral manifestations in two cohorts of women in San Francisco.J Acquir Immune D efic Syndr 1994;7:964-971.

21. Melnick SL, Engel D, Truelove E, D e Rouen T, Morton T, Schubert M et al. 0 ral mucosal lesions:Association with the presence of antibodies to the human immunodeficiency virus. 0 ral Surg 0 ral Med 0 ral Pathol 1989;68:37-43
22. Begg MD, Lamster IB, Panageas KS, Mitchell-Lewis D, Phelan JA, G rbic JT.A prospective study of oral lesions and their predictive value for progression of HIV disease. O ral D is 1997;3:176-183.

23. Begg MD, Panageas KS, Mitchell-Lewis D, Bucklan RS, Phelan JA, Lamster IB. 0 ral lesions as markers of severe immunosupresion in HIV-infected homosexual men and injection drug users. 0 ral Surg 0 ral Med 0 ral Pathol 0 ral Radiol Endod 1996;82:276-283.

24. G lick M, Muzyka BC, Lurie D, Salkin LM. 0 ral manifestations associated with HIV-related disease as markers for immune suppression and AIDS. O ral Surg 0 ral Med 0 ral Pathol 0 ral Radiol Endod 1994;77: 344-349. 